\title{
Corrigendum: Functional variants in the B-cell gene BANK1 are associated with systemic lupus erythematosus
}

Sergey V Kozyrev, Anna-Karin Abelson, Jerome Wojcik, Ammar Zaghlool, MV Prasad Linga Reddy, Elena Sanchez, Iva Gunnarsson, Elisabet Svenungsson, Gunnar Sturfelt, Andreas Jönsen, Lennart Truedsson, Bernardo A Pons-Estel, Torsten Witte, Sandra D’Alfonso, Nadia Barrizzone, Maria Giovanna Danieli, Carmen Gutierrez, Ana Suarez, Peter Junker, Helle Laustrup, Maria Francisca González-Escribano, Javier Martin, Hadi Abderrahim \& Marta E Alarcón-Riquelme

Nature Genetics 40, 211-216 (2008); published online 20 January 2008; corrected after print 27 March 2008

In the version of this article initially published, the name of the 15th author was misspelled. The correct author name is Nadia Barizzone. Also, the affiliation of Javier Martin was incomplete. Dr. Martin is affiliated with Instituto de Biomedicina López-Neyra, Grenada 18100, Spain and Consejo Superior de Investigaciones Científicas (CSIC), Grenada 18100, Spain. The errors have been corrected in the HTML and PDF versions of the article. 